\title{
LRRK2 mediated Rab8a phosphorylation promotes lipid storage
}

\author{
Miao $\mathrm{Yu}^{1 \dagger}$, Muhammad Arshad ${ }^{2 \dagger}$, Wenmin Wang $^{1 \dagger}$, Dongyu Zhao ${ }^{1}$, Li Xu ${ }^{1}$ and Linkang Zhou ${ }^{1 *}$
}

\begin{abstract}
Background: Several mutations in leucine rich repeat kinase 2 (LRRK2) gene have been associated with pathogenesis of Parkinson's disease (PD), a neurodegenerative disorder marked by resting tremors, and rigidity, leading to Postural instability. It has been revealed that mutations that lead to an increase of kinase activity of LRRK2 protein are significantly associated with PD pathogenesis. Recent studies have shown that some Rab GTPases, especially Rab8, serve as substrates of LRRK2 and undergo phosphorylation in its switch II domain upon interaction. Current study was performed in order to find out the effects of the phosphorylation of Rab8 and its mutants on lipid metabolism and lipid droplets growth.
\end{abstract}

Methods: The phosphorylation status of Rab8a was checked by phos-tag gel. Point mutant construct were generated to investigate the function of Rab8a. 3T3L1 cells were transfected with indicated plasmids and the lipid droplets were stained with Bodipy. Fluorescent microscopy experiments were performed to examine the sizes of lipid droplets. The interactions between Rab8a and Optineurin were determined by immunoprecipitation and western blot.

Results: Our assays demonstrated that Rab8a was phosphorylated by mutated LRRK2 that exhibits high kinase activity. Phosphorylation of Rab8a on amino acid residue T72 promoted the formation of large lipid droplets. T72D mutant of Rab8a had higher activity to promote the formation of large lipid droplets compared with wild type Rab8a, with increase in average diameter of lipid droplets from $2.10 \mu \mathrm{m}$ to $2.46 \mu \mathrm{m}$. Moreover, phosphorylation of Rab8a weakened the interaction with its effector Optineurin.

Conclusions: Y1699C mutated LRRK2 was able to phosphorylate Rab8a and phosphorylation of Rab8a on site 72 plays important role in the fusion and enlargement of lipid droplets. Taken together, our study suggests an indirect relationship between enhanced lipid storage capacity and PD pathogenesis.

Keywords: LRRK2, Rab8a, Lipid droplets, Lipid storage, Parkinson's disease

\section{Background}

Rabs are a group of about 70 eukaryotic proteins located on different membranes that play important roles in all stages of vesicle trafficking including budding, motility and fusion $[1,2]$. The most important feature of Rab proteins is that they can switch from inactive GDP-bound form to active GTP-bound form and recruit unique effectors which play their roles in vesicle trafficking [3]. The binding of GTP or GDP changes the conformation in Rab Switch I and Switch II zones which are crucial for maintaining the specific conformation of Rab proteins. The

\footnotetext{
* Correspondence: zhoulinkang@tsinghua.edu.cn

${ }^{\dagger}$ Equal contributors

${ }^{1}$ MOE Key Laboratory of Bioinformatics and Tsinghua-Peking Center for Life Sciences, School of Life Sciences, Tsinghua University, Beijing 100084, China Full list of author information is available at the end of the article
}

switching of Rab GTPases is mediated by four classes of proteins and the inhibition of Rab pathway results in diseases like immunodeficiency or neurological disorders [4]. Many Rabs can undergo phosphorylation on serine or threonine residues [5]. Rab5a has been reported to be phosphorylated by PKC (Protein kinase C) on Thr (threonine)-7 site. Rab5a phosphorylation is functionally necessary for Rac1 activation, actin rearrangement and T-cell motility [6]. Rab24 can undergo tyrosine phosphorylation and this modification may influence Rab24 targeting and interactions with effector protein complexes [7].

Rab8 has shown to play important roles in membrane trafficking. It plays irreplaceable roles in multiple biological processes including cellular morphology, cell polarity, cell movement, neural differentiation and ciliogenesis [8]. Knocking down of Rab8 or its effector Optineurin results 
in the inhibition of transportation of transferrin protein from membrane to intracellular part while transferrin protein receptor fails to return to ERC (endocytic recycling compartment) [9, 10]. Furthermore, Rab8 is recruited by Rab6 onto the vesicles responsible for exocytosis where it controls exocytosis activity $[11,12]$. Rab8 could also regulate the fusion between vesicles and cell membrane [13].

LRRK2 (leucine rich repeat kinase 2) has been a hot spot in the field of neurology as its mutations have been associated with Parkinson's disease (PD) [14, 15]. PD is a common neurodegenerative disease affecting approximately $1 \%$ of the elderly population and is marked by resting tremors, rigidity, and akinesia $[16,17]$. The progression of the disease results in postural instability. While the causative mechanisms of PD remain elusive, the findings that more than twenty mutations on $L R R K 2$ gene are related to PD have brought LRRK2 into main focus of research [18]. LRRK2 is a large protein consisting of central kinase and GTPase domains which are surrounded by several proteinprotein interaction regions. PD pathogenic LRRK2 mutations (G2019S, I2020T, R1441C/G/H, Y1699C) are primary causes for PD pathogenesis. Till now, it remains unclear how LRRK2 mutations occurring in different functional domains predispose to PD. The most powerful PD-associated LRRK2 mutation is G2019S, which increases LRRK2 kinase activity two to three fold $[19,20]$. Since LRRK2 is a kinase, its substrates may have important role in pathogenesis.

Recently, a subset of Rab GTPases has been identified as key LRRK2 substrates. Through a combination of phosphoproteomics and molecular genetics approaches, it has been proved that several Rab GTPases especially Rab8 can be phosphorylated by LRRK2 on an evolutionary conserved residue in switch II domain [21]. Since switch II domain plays crucial role in maintaining Rab protein conformation, the phosphorylation may alter the behavior of Rab proteins. Considering this, we have tried to find more evidences about the consequences of the phosphorylation of Rab8 and have found out an important relationship between lipid storage and PD pathogenesis.

\section{Methods}

\section{Cell culture}

Two hundred ninety-three $\mathrm{T}$ cells and 3 T3-L1 preadipocytes were cultured in DMEM (Invitrogen, USA) containing 10\% FBS (Invitrogen, USA), 2 mM L-glutamine, $100 \mathrm{U} / \mathrm{mL}$ penicillin and $100 \mu \mathrm{g} / \mathrm{ml}$ streptomycin at $37{ }^{\circ} \mathrm{C}$ in humidified incubators containing $5 \% \mathrm{CO}_{2}$. Methods for 3T3L1 preadipocytes differentiation and electrotransfection were similar as previously described [22].

\section{Cell transfection}

DNA plasmids were transfected into $293 \mathrm{~T}$ cells and 3 T3-L1 pre-adipocytes using Lipofectamine 2000 following manufacturer's instructions (Invitrogen, USA).

\section{Plasmids construction}

Human LRRK2 and its mutant (LRRK2 Y1699C) plasmids were purchased from Addgene (code \#17609 and \#25364). Full-length cDNAs encoding mouse Rab8a and Optinurin were amplified from cDNA of 3 T3-L1 adipocytes. These cDNAs were subcloned into pCMV5-HA or pCMV5FLAG using specific restriction sites. Point mutations of Rab8a were cloned by a PCR-based site-directed mutagenesis method (Stratagene, USA). The accuracy of each plasmid DNAs was tested by sequence analysis.

\section{Western blot}

Methods for immunoprecipitation and western blot sample preparation were same as previously described [22]. Anti-FLAG M2 agarose beads (cat: A2220) were purchased from Sigma. The antibody against Fsp27 was used as described previously [22]. Antibodies against Actin (A5441, Sigma), FLAG (mouse source, F1804, sigma), MYC (sc-40, Santa Cruz), LRRK2 (5559, Cell signaling), Rab8a (6975, Cell signaling) and HA (sc-7392, Santa Cruz) were used for western blot analysis. The blots were detected using HRP-conjugated secondary antibodies (GE Healthcare, UK) and the ECL-Plus system.

\section{Phos-tag gel}

Phos-tag acrylamide and $\mathrm{MnCl}_{2}$ were added to a standard gel solution to the final concentration of $50 \mathrm{mM}$ and $100 \mathrm{mM}$, respectively. Then gels were polymerized by ammonium per-sulfate and TEMED. Cell lysates used for Phos-tag SDS-PAGE were supplemented with $\mathrm{MnCl}_{2}$ at $10 \mathrm{mM}$ to neutralize the effect of EDTA in the lysates. After running SDS-PAGE, gels were washed 3 times with transfer buffer containing $10 \mathrm{mM}$ EDTA followed by a wash with transfer buffer (10 min each). Blotting to nitrocellulose membranes was carried out by following a standard protocol. Phos-tag acrylamide was kept at $4{ }^{\circ} \mathrm{C}$ in black tubes blocking out light because Phos-tag acrylamide is light-sensitive.

\section{Imaging}

Images for analyzing lipid droplets sizes were acquired under an LSM710 confocal microscope (Carl Zeiss) with $63 \times$ oil immersion objective. Images were exported out in 16-bit TIFF format. Further processing of single images (e.g., amplifying a certain region) was performed in Photoshop (CS2; Adobe).

\section{Lipid droplet size analysis}

Quantitative analysis of LD size in 3 T3-L1 preadipocytes was performed as described by Sun et al. [23]. For quantitative analysis of cells containing large LDs, DNA plasmids were transfected into $3 \mathrm{~T} 3-\mathrm{L} 1$ preadipocytes using Lipofectamine 2000. Cells were incubated with $200 \mathrm{mM}$ OA complexed with albumin for $15 \mathrm{~h}$ before 
they were fixed. The largest LDs' sizes were measured in each cell. Approximately 150 cells from three independent experiments were analyzed. Images were obtained under an inverted microscope Axiovert.

\section{Statistics}

All statistical analyses were performed in GraphPad Prism Version 5 (GraphPad Software). Significance was established using a two-tailed Student's $t$-test. Differences were considered significant at $P<0.05$. **** indicated $P<0.001$.

\section{Results}

\section{Rab8a is phosphorylated by LRRK2}

In order to confirm whether Rab8a could be phosphorylated by LRRK2, we overexpressed FLAG-tagged Rab8a (Wild-type form and T72A form. "T" represent threonine and "A" represent alanine) and MYC-tagged LRRK2 (Wild-type form and Y1699C form) in 293 T cells. The phosphorylation form of Rab8a was detected using phos-tag gel. As shown in Fig. 1a, Y1699C mutation of LRRK2, but not wild-type LRRK2, could phosphorylate Rab8a as indicated by the shift in the band. The T72A mutant of Rab8a could not be phosphorylated by LRRK2 (Y1699C), indicating that the phosphorylation of Rab8a by LRRK2 Y1699C exclusively occurs on T72 site. Next, we wondered whether endogenous Rab8a could be phosphorylated by LRRK2. We first checked the expression level of Rab8a and LRRK2 in differentiated adipocytes. The expression of Rab8a was found to be higher compared with that in brain. The expression of LRRK2 was detected in adipocytes, although the expression level was low compared with that in brain (Fig. 1b). We transfected LRRK2 (Y1699C) in differentiated adipocytes. Then we performed immunoprecipitation of Rab8a using Rab8a antibody, and later ran the phos-tag gel to check the phosphorylation status of Rab8a. Overexpression of LRRK2 in adipocytes could dramatically induce the phosphorylation of endogenous Rab8a (Fig. 1c).

\section{Phosphorylated Rab8a on site $\mathbf{7 2}$ promotes lipid droplets fusion and enlargement}

Our previous data has shown that Rab8a plays important role in Fsp27 mediated lipid droplet fusion and enlargement [22]. Here, we wanted to investigate the role of phosphorylated Rab8a on lipid droplet enlargement. We overexpressed Rab8a (wild-type, T72A), LRRK2 (wild-type, Y1699C) together with Fsp27-Cherry and relative controls in $3 \mathrm{~T} 3-\mathrm{L} 1$ pre-adipocytes and the lipid droplets in the cells were stained with Bodipy 493/503. Overexpression of Rab8a, LRRK2 (Y1699C) could not enhance the size of lipid droplets compared with the controls. However, overexpression of Fsp27 could dramatically induce lipid droplets' size compared with control cells. Rab8a could enhance Fsp27's ability in promoting

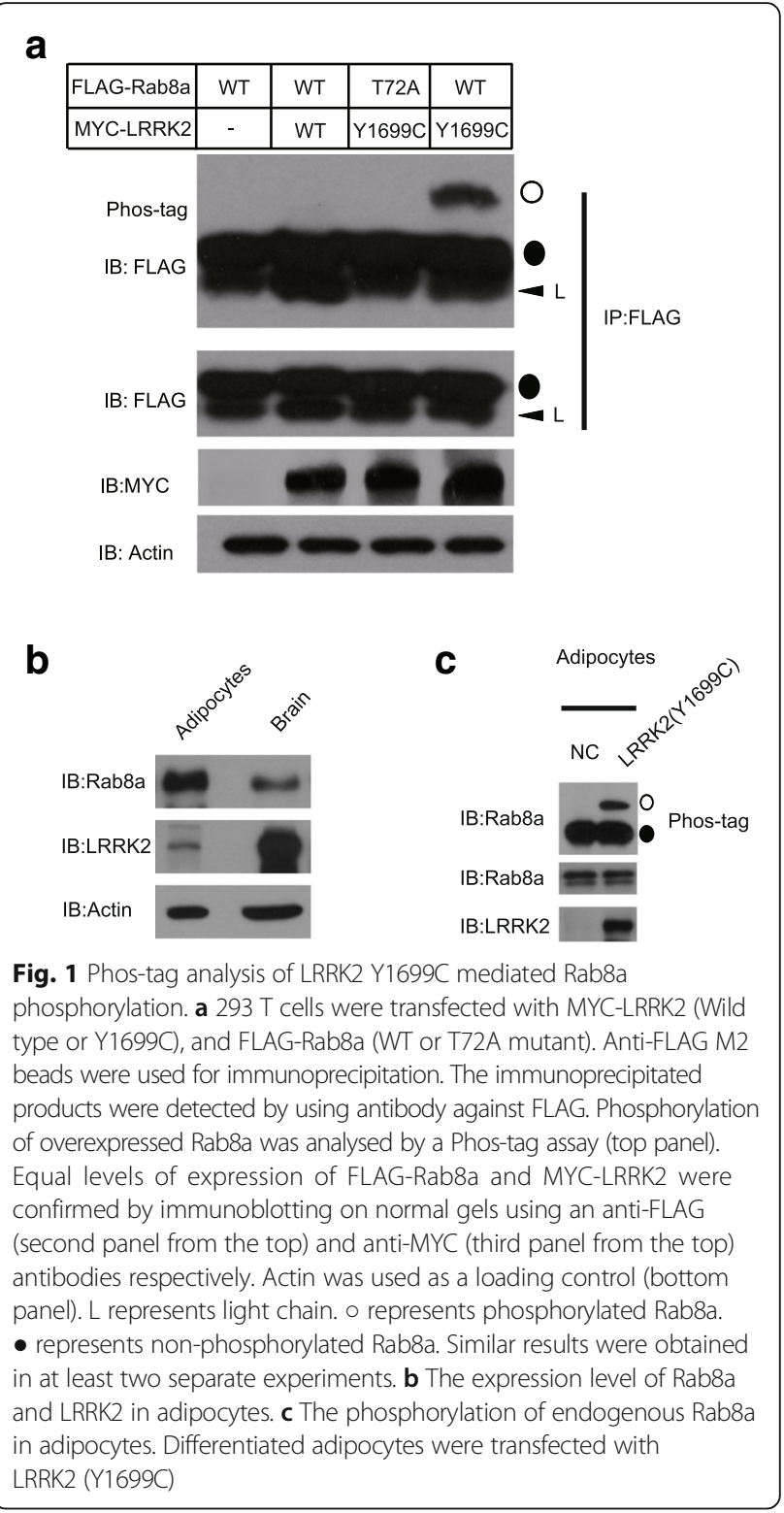

lipid droplets enlargement. Co-overexpression of LRRK2 (Y1699C) and Rab8a resulted in the formation of enlarged lipid droplets compared with Rab8a overexpression alone (Fig. 2a-d), with increase in average diameter of lipid droplets from $2.26 \mu \mathrm{m}$ to $2.69 \mu \mathrm{m}$. However, co-expression of wild-type LRRK2 with Rab8a showed no significant impact on the size of lipid droplets when compared with Rab8a overexpression alone. The size of lipid droplets in cells overexpressing Rab8a (T72A) together with LRRK2 (Y1699C) was similar with the cells overexpressing Rab8a alone. These data indicate that the phosphorylation of Rab8a on site 72 plays important role in the fusion and enlargement of lipid droplets. 

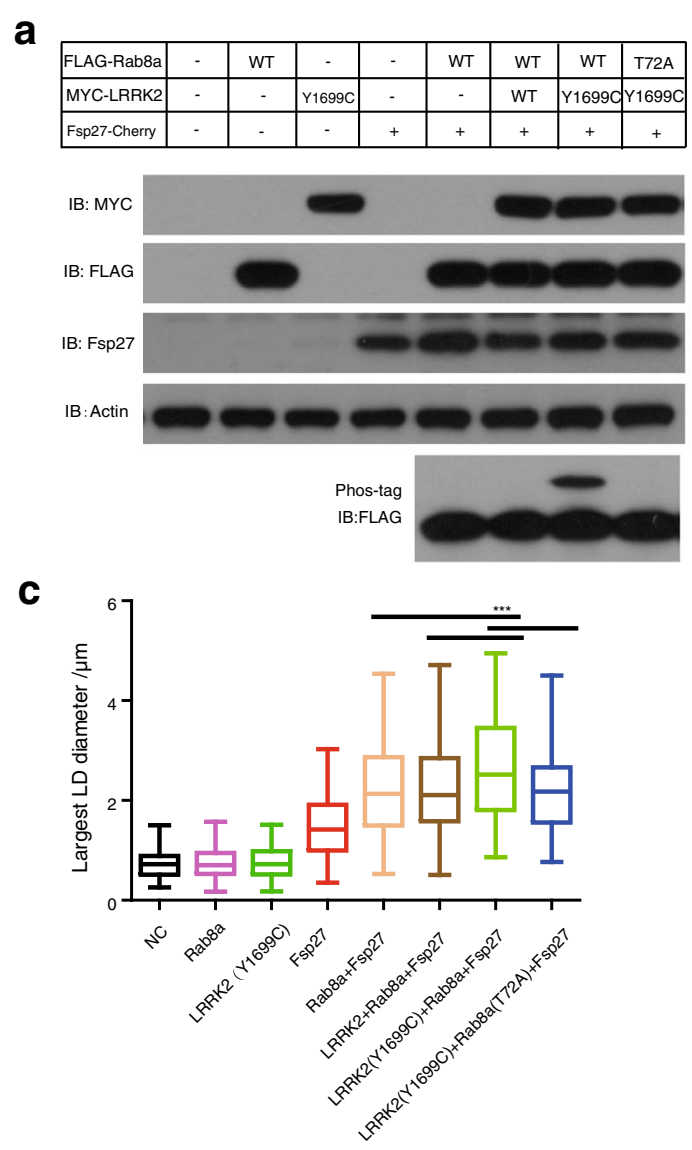

d

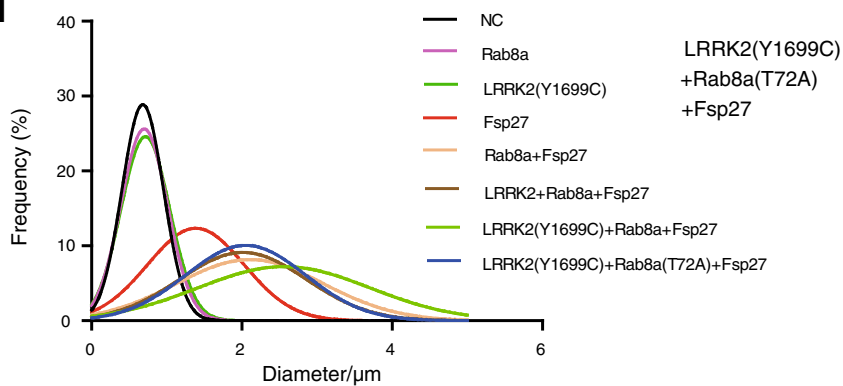

b

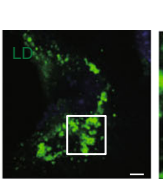

Enlarge

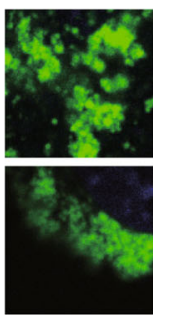

LRRK2(Y1699C)
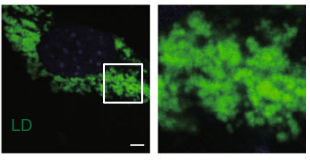

Fsp27
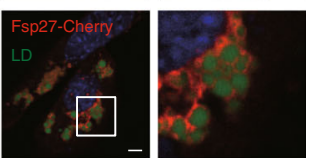

Rab8a
+ Fsp27
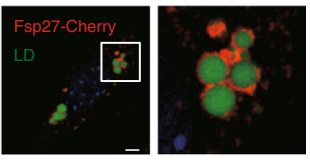

LRK2

+ Rab8a

+ Fsp27
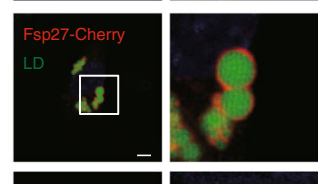

LRRK2(Y1699C) + Rab8a +Rab8a
+ Fsp27
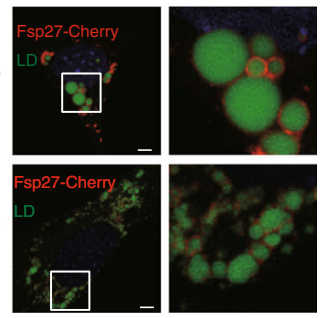

Fig. 2 Rab8a phosphorylation promotes lipid droplets enlargement. 3 T3-L1 pre-adipocytes were transfected with MYC-LRRK2 (Wild type or Y1699C), FLAG-Rab8a (WT or T72A mutant) and Fsp27-Cherry. Oleic acid was added to promote the formation of LDs for 15 h. LDs were labeled with Bodipy 493/503 (Green). a Expression levels of LRRK2, Rab8a and Fsp27 were detected by western blot. b Co-expression of LRRK2 Y1699C with FLAG-Rab8a increases the sizes of LDs when over-expressing Fsp27. Scale bars represent $20 \mu \mathrm{m}$. c-d Statistical analysis of (b). Significance was established using a two-tailed Student's t-test. Differences were considered significant at $P<0.05{ }^{* * *} P<0.001$

\section{T72D/E form of Rab8a promotes the formation of large} lipid droplets

Since point mutation from threonine (T) to aspartic acid (D) or glutamic acid (E) can mimic phosphorylation, different mutations on the T72 site of Rab8a were generated (T72A, T72D and T72E). T72A represents the nonphosphorylated form of Rab8a while T72D/E mimics the phosphorylated form of Rab8a. We wanted to investigate the role of above mentioned Rab8a mutants in lipid droplets enlargement. Overexpression of WT Rab8a could enhance Fsp27's ability to promote the formation of large lipid droplets. Our results showed that overexpression of Rab8a (T72D) and Rab8a (T72E) increased the size of LDs from $2.27 \mu \mathrm{m}$ to $2.87 \mu \mathrm{m}$ when compared with overexpression of Rab8a or Rab8a (T72A) (Fig. 3). According to our previous findings [22], the GDP-bound form of Rab8a (T22 N) could significantly enhance the formation of large lipid droplets as compared to wild-type Rab8a. Interestingly, the effects of T72D and T72E resemble the effect of Rab8a 


\section{a}

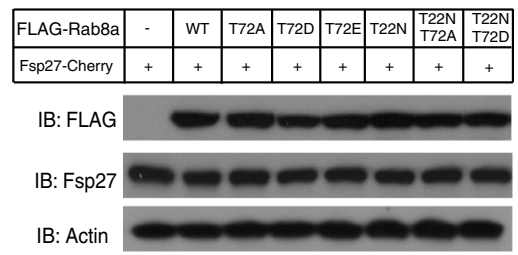

C

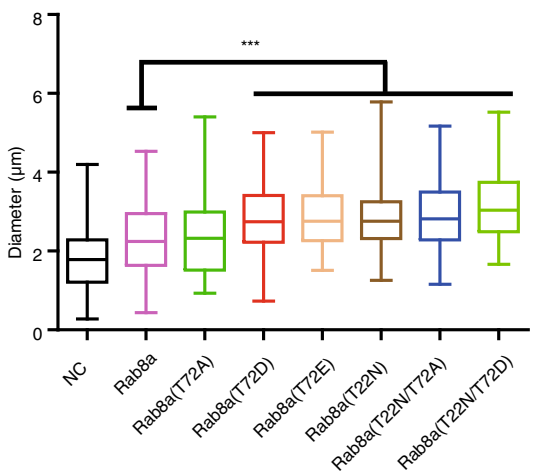

d

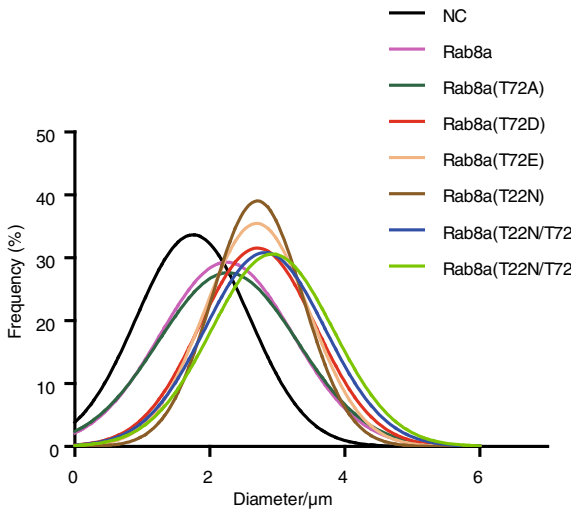

b

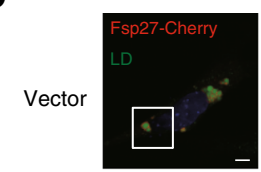

Enlarge

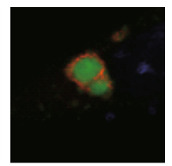

Rab8a
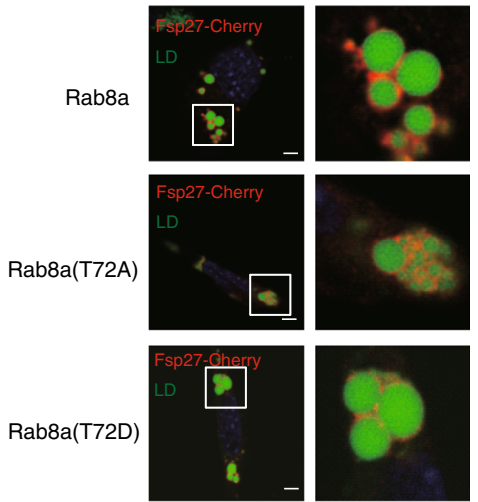

Rab8a(T72E)
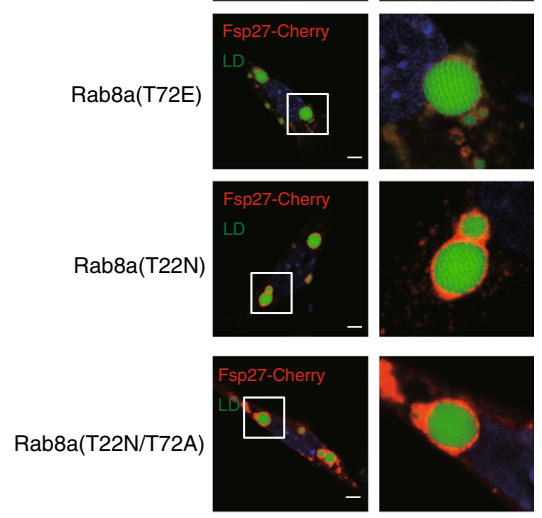

Rab8a(T22N/T72D)
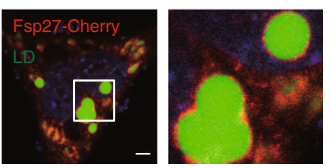

Fig. 3 Overexpression of mimic phosphorylated Rab8a (Rab8a T72D and Rab8a T72E) increases the size of LDs. 3T3L1 pre-adipocytes were transfected with FLAG-Rab8a (WT, T72A, T72D, T72E, T22 N, T22 N/T72A, T22 N/T72D) and Fsp27-Cherry. Oleic acid was added to promote the formation of LDs for 15 h. LDs were labeled with Bodipy 493/503 (Green). a Western blots showing protein levels of FLAG-tagged Rab8a and its mutations. b \& c Overexpression of Rab8a T72D and T72E increases the sizes of LDs under the circumstances of over-expressing Fsp27 while overexpression of wild-type Rab8a or phosphorylation defective Rab8a (T72A) shows no such effects. Scale bars represent 20 mm. d Statistical analysis of (b). Significance was established using a two-tailed Student's t-test. Differences were considered significant at $P<0.05$. ${ }^{* * *} P<0.001$

(T22 N), indicating that the phosphorylation of Rab8a on site 72 may affect the GTP/GDP binding status of Rab8a. We then introduced T22 N/T72A and T22 N/T72D, two double mutants of Rab8a. The T22 N/T72A mutant demonstrated effect similar to that of T22 $\mathrm{N}$ on the size of lipid droplets. The T22 N/T72D mutant showed slightly increased effect in promoting lipid droplets enlargement when compared with $\mathrm{T} 22 \mathrm{~N}$, with an increase in size of lipid droplets from $2.84 \mu \mathrm{m}$ to $3.17 \mu \mathrm{m}$.

Next, we investigated whether the phosphorylation of Rab8a on 72 site affects its GTP/GDP binding status. Optineurin has been reported to be the effector of Rab8a which binds to the GTP form but not to the GDP form of Rab8a [9]. We co-expressed FLAG-Rab8a and its different mutations (containing wild-type, T72A, T72D, T72E, T22 N, T22 N/T72A, T22 N/T72D) together with HA-Optineurin, then immunoprecipitated down FLAGtagged Rab8a. As shown in Fig. 4, Rab8a-T22 N showed nearly no interaction with Optineurin compared with wild-type Rab8a. Interestingly, Rab8a-T72D and Rab8aT72E also showed weak interactions with Optineurin which resembles the Rab8a-T22 N interaction. T22 N/ T72A and T22 N/T72D mutants also lost the binding with Optineurin. These results show that phosphorylation of Rab8a weakens its interaction with its effector Optineurin. 


\begin{tabular}{|c|c|c|c|c|c|c|c|c|}
\hline FLAG-Rab8a & - & WT & T72A & T72D & T72E & T22N & $\begin{array}{c}\text { T22N } \\
\text { T72A }\end{array}$ & $\begin{array}{c}\text { T22N } \\
\text { T72D }\end{array}$ \\
\hline HA-Optn & + & + & + & + & + & + & + & + \\
\hline
\end{tabular}

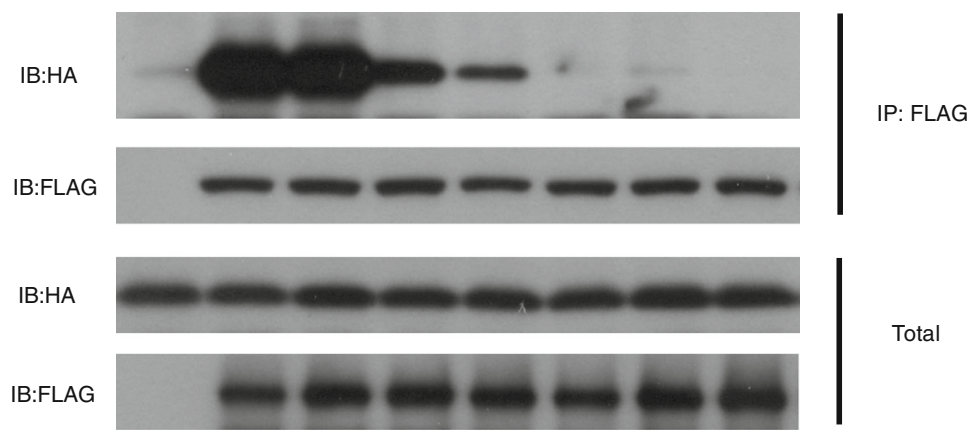

Fig. 4 Phosphorylation of Rab8a weakens its interaction with its effector Optineurin. Two hundred ninety-three T cells were transfected with FLAG-Rab8a (WT, T72A, T72D, T72E, T22 N, T22 N/T72A, T22 N/T72D) and HA-Optineurin. Anti-FLAG M2 beads were used for immunoprecipitation. The immunoprecipitated products were detected by using antibodies against FLAG or HA. IP, immunoprecipitation; IB, immunoblot; Western blots showing protein level of HA-Optineurin (top panel) and FLAG-Rab8a (second panel from the top) in the IP products. Western blots showing protein level of HA-Optineurin (third panel from the top) and FLAG-Rab8a (bottom panel) in the total cell lysis

\section{Discussion}

In this study, we have shown that Rab8a could be phosphorylated by Y1699C mutated LRRK2. Previous studies showed that there had been an increase in enzyme activity of LRRK2 Y1699C compared with WT LRRK2 [20, 24, 25]. The Rab8a 72 Tyrosine was the only site phosphorylated by mutated LRRK2. We also found out that T72 phosphorylation significantly enhanced the lipid storage activity of Rab8a. Our previous work has already shown that GDP form of Rab8a (T22 N) has higher activity in promoting lipid storage [22]. T72D/E mutation stabilizes the GDP form of Rab8a.

Earlier studies have shown that LRRK2 mutations (Y1699C, G2019S) which increase the activity of LRRK2 are the most common causes of the inherited form of PD pathogenesis [26], however, it is still unclear why overactivation of LRRK2 is harmful and what role it plays inside cells. Moreover, LRRK2 knockout models studies have manifested Rabs as primary substrates of LRRK2 as LRRK2 knockout rats and mice have shown deformed lungs and kidneys due to possible defects in the autophagosome pathway regulated by activated Rab proteins [27]. Recent studies have shown that both Rab8a and Rab10 are the substrates of LRRK2, indicating that LRRK2-Rabs circuit plays important role in the pathogenesis of PD.

Overexpression of Rab8a, Rab1 and Rab3a proteins alleviate PD through reduced $\alpha$-synuclein-induced cytotoxicity $[28,29]$. As $\alpha$-synuclein is a lipid droplet bound protein, its dysfunction or overexpression can cause PD as well as lipid droplet formation [30-32]. Some recent studies have identified the presence of lipid droplets in neurons and in glia under certain disease conditions which suggest some association of disrupted lipid-droplet function with neurodegeneration [33, 34]. The size of LDs reflects lipid storage capacity and has been linked to the development of obesity, diabetes, hepatic steatosis and atherosclerosis [35]. The LRRK2-Rab8a mediated LDs enlargement may play important role in PD pathogenesis. Other kinases found mutated in PD pathogenesis, such as PINK1, could also regulate the phosphorylation of Rab8a at site Serine 111 [36]. Some studies have begun focusing on the role of lipid metabolism in PD. A recent study has demonstrated that many sphingolipid, glycerophospholipid and cholesterol species were found altered in the visual cortex of PD patients [37, 38]. Here, we have proposed that Rab8a phosphorylation by LRRK2 alters the ability of lipid storage in PD. Normalization of lipid metabolism may present a novel route for treatment of some types of PD patients.

Another study has established that change in Rab homeostasis may contribute towards development of PD. As LRRK2 is the main regulator of Rab proteins, overactive LRRK2 results in increased Rab phosphorylation, altering the balance between cytosolic and membrane bound Rab, which in turns disturbs intracellular trafficking. In PD-associated LRRK2 mutations, the membrane-cytosol balance of Rabs is shifted towards the membrane causing accumulation of inactive Rabs in the membranes [21]. Moreover, pathogenic LRRK2 mutations outside the kinase domain can also increase Rab phosphorylation which, according to our study, would result in the formation of enlarged lipid droplets, a manifestation of PD pathogenesis.

\section{Conclusion}

Our findings have proved that Y1699C mutated LRRK2, but not wild-type LRRK2, could phosphorylate Rab8a 
and that phosphorylation of Rab8a on site 72 plays important role in the fusion and enlargement of lipid droplet. Furthermore, our results show that phosphorylation of Rab8a weakens its interaction with its effector Optineurin. It will be interesting to investigate the direct role of lipid droplets accumulation with pathogenesis of PD in order to explore therapeutic interventions against this disease.

\section{Abbreviations}

ERC: Endocytic recycling compartment; LDs: Lipid droplets; LRRK2: Leucine rich repeat kinase 2; PD: Parkinson's disease; PKC: Protein kinase $C$

\section{Acknowledgements}

We would like to thank the members of the Peng Li's Laboratory at Tsinghua University for their helpful discussions.

\section{Funding}

LZ was supported by the Young Elite Scientists Sponsorship Program By CAST (China Association for Science and Technology). This work was supported by grants from the National Natural Science Foundation of China (31771303 to LZ) and from the China Postdoctoral Science Foundation (2012 M520249 and 2013 T60103 to LZ).

\section{Availability of data and materials}

Data are available on request to the authors.

\section{Authors' contributions}

MY, MA, WW and LZ conceived and designed the experiments. DZ and LX provided some materials and analyzed the data. MA and LZ wrote the paper. All authors read and approved the final manuscript.

\section{Ethics approval and consent to participate}

Not applicable.

\section{Consent for publication}

Not applicable.

\section{Competing interests}

The authors declare that they have no competing interest.

\section{Publisher's Note}

Springer Nature remains neutral with regard to jurisdictional claims in published maps and institutional affiliations.

\footnotetext{
Author details

'MOE Key Laboratory of Bioinformatics and Tsinghua-Peking Center for Life Sciences, School of Life Sciences, Tsinghua University, Beijing 100084, China. ${ }^{2}$ Department of Bioinformatics and Biotechnology, International Islamic University, Islamabad 44000, Pakistan.
}

Received: 23 August 2017 Accepted: 19 February 2018 Published online: 27 February 2018

\section{References}

1. Stenmark H. Rab GTPases as coordinators of vesicle traffic. Nat Rev Mol Cell Biol. 2009;10:513-25.

2. Kelly EE, Horgan CP, Goud B, McCaffrey MW. The Rab family of proteins: 25 years on. Biochem Soc Trans. 2012;40:1337-47.

3. Soldati T, Shapiro AD, Svejstrup AB, Pfeffer SR. Membrane targeting of the small GTPase Rab9 is accompanied by nucleotide exchange. Nature. 1994;369:76-8

4. Seabra MC, Mules EH, Hume AN. Rab GTPases, intracellular traffic and disease. Trends Mol Med. 2002;8:23-30.

5. Overmeyer JH, Maltese WA. Tyrosine phosphorylation of Rab proteins. Methods Enzymol. 2005:403:194-202.

6. Ong ST, Freeley M, Skubis-Zegadlo J, Fazil MH, Kelleher D, Fresser F, Baier G, Verma NK, Long A. Phosphorylation of Rab5a protein by protein kinase $\mathrm{C}$ is crucial for T-cell migration. J Biol Chem. 2014;289:19420-34.
7. Ding J, Soule G, Overmeyer JH, Maltese WA. Tyrosine phosphorylation of the Rab24 GTPase in cultured mammalian cells. Biochem Biophys Res Commun. 2003;312:670-5.

8. Peranen J. Rab8 GTPase as a regulator of cell shape. Cytoskeleton (Hoboken). 2011;68:527-39.

9. Park B, Ying H, Shen X, Park JS, Qiu Y, Shyam R, Yue BY. Impairment of protein trafficking upon overexpression and mutation of optineurin. PLoS One. 2010;5:e11547.

10. Nagabhushana A, Chalasani ML, Jain N, Radha V, Rangaraj N, Balasubramanian D, Swarup G. Regulation of endocytic trafficking of transferrin receptor by optineurin and its impairment by a glaucomaassociated mutant. BMC Cell Biol. 2010;11:4.

11. Grigoriev I, Yu KL, Martinez-Sanchez E, Serra-Marques A, Smal I, Meijering E, Demmers J, Peranen J, Pasterkamp RJ, van der Sluijs P, et al. Rab6, Rab8, and MICAL3 cooperate in controlling docking and fusion of exocytotic carriers. Curr Biol. 2011;21:967-74.

12. Dong C, Yang L, Zhang X, Gu H, Lam ML, Claycomb WC, Xia H, Wu G. Rab8 interacts with distinct motifs in alpha2B-and beta2-adrenergic receptors and differentially modulates their transport. J Biol Chem. 2010;285:20369-80

13. Bond LM, Peden AA, Kendrick-Jones J, Sellers JR, Buss F. Myosin VI and its binding partner optineurin are involved in secretory vesicle fusion at the plasma membrane. Mol Biol Cell. 2011;22:54-65.

14. Paisan-Ruiz C, Jain S, Evans EW, Gilks WP, Simon J, van der Brug M, Lopez de Munain A, Aparicio S, Gil AM, Khan N, et al. Cloning of the gene containing mutations that cause PARK8-linked Parkinson's disease. Neuron. 2004;44:595-600.

15. Zimprich A, Biskup S, Leitner $P$, Lichtner $P$, Farrer M, Lincoln S, Kachergus J, Hulihan M, Uitti RJ, Calne DB, et al. Mutations in LRRK2 cause autosomaldominant parkinsonism with pleomorphic pathology. Neuron. 2004;44:601-7.

16. Halliday G, Lees A, Stern M. Milestones in Parkinson's disease-clinical and pathologic features. Mov Disord. 2011;26:1015-21.

17. Lees AJ, Hardy J, Revesz T. Parkinson's disease. Lancet. 2009;373:2055-66.

18. West AB, Moore DJ, Biskup S, Bugayenko A, Smith WW, Ross CA, Dawson VL, Dawson TM. Parkinson's disease-associated mutations in leucine-rich repeat kinase 2 augment kinase activity. Proc Natl Acad Sci U S A. 2005;102: $16842-7$.

19. Greggio E, Jain $S$, Kingsbury A, Bandopadhyay R, Lewis $P$, Kaganovich A, van der Brug MP, Beilina A, Blackinton J, Thomas KJ, et al. Kinase activity is required for the toxic effects of mutant LRRK2/dardarin. Neurobiol Dis. 2006:23:329-41.

20. Gloeckner CJ, Kinkl N, Schumacher A, Braun RJ, O'Neill E, Meitinger T, Kolch W, Prokisch $\mathrm{H}$, Ueffing M. The Parkinson disease causing LRRK2 mutation I2020T is associated with increased kinase activity. Hum Mol Genet. 2006;15: 223-32.

21. Steger $\mathrm{M}$, Tonelli $\mathrm{F}$, Ito $\mathrm{G}$, Davies $\mathrm{P}$, Trost $\mathrm{M}$, Vetter $\mathrm{M}$, Wachter $\mathrm{S}$, Lorentzen E, Duddy G, Wilson S, et al. Phosphoproteomics reveals that Parkinson's disease kinase LRRK2 regulates a subset of Rab GTPases. elife. 2016;5:e12813.

22. Wu L, Xu D, Zhou L, Xie B, Yu L, Yang H, Huang L, Ye J, Deng H, Yuan YA, et al. Rab8a-AS160-MSS4 regulatory circuit controls lipid droplet fusion and growth. Dev Cell. 2014;30:378-93.

23. Sun Z, Gong J, Wu H, Xu W, Wu L, Xu D, Gao J, Wu JW, Yang H, Yang M, Li P. Perilipin1 promotes unilocular lipid droplet formation through the activation of Fsp27 in adipocytes. Nat Commun. 2013:4:1594.

24. Jaleel M, Nichols RJ, Deak M, Campbell DG, Gillardon F, Knebel A, Alessi DR. LRRK2 phosphorylates moesin at threonine-558: characterization of how Parkinson's disease mutants affect kinase activity. Biochem J. 2007;405:307-17.

25. Daniels V, Vancraenenbroeck R, Law BM, Greggio E, Lobbestael E, Gao F, De Maeyer M, Cookson MR, Harvey K, Baekelandt V, Taymans JM. Insight into the mode of action of the LRRK2 Y1699C pathogenic mutant. J Neurochem. 2011;116:304-15.

26. Healy DG, Falchi M, O'Sullivan SS, Bonifati V, Durr A, Bressman S, Brice A, Aasly J, Zabetian CP, Goldwurm S, et al. Phenotype, genotype, and worldwide genetic penetrance of LRRK2-associated Parkinson's disease: a case-control study. Lancet Neurol. 2008;7:583-90.

27. Baptista MA, Dave KD, Frasier MA, Sherer TB, Greeley M, Beck MJ, Varsho JS, Parker GA, Moore C, Churchill MJ, et al. Loss of leucine-rich repeat kinase 2 (LRRK2) in rats leads to progressive abnormal phenotypes in peripheral organs. PLoS One. 2013;8:e80705.

28. Cooper AA, Gitler AD, Cashikar A, Haynes CM, Hill K, Bhullar B, Liu K, Xu K, Strathearn KE, Liu F, et al. Alpha-synuclein blocks ER-Golgi traffic and Rab1 rescues neuron loss in Parkinson's models. Science. 2006;313:324-8. 
29. Gitler AD, Bevis BJ, Shorter J, Strathearn KE, Hamamichi S, Su LJ, Caldwell KA, Caldwell GA, Rochet JC, JM MC, et al. The Parkinson's disease protein alphasynuclein disrupts cellular Rab homeostasis. Proc Natl Acad Sci U S A. 2008:105:145-50.

30. Cole NB, Murphy DD, Grider T, Rueter S, Brasaemle D, Nussbaum RL. Lipid droplet binding and oligomerization properties of the Parkinson's disease protein alpha-synuclein. J Biol Chem. 2002;277:6344-52.

31. Wang S, Horn PJ, Liou LC, Muggeridge MI, Zhang Z, Chapman KD, Witt SN . A peroxisome biogenesis deficiency prevents the binding of alphasynuclein to lipid droplets in lipid-loaded yeast. Biochem Biophys Res Commun. 2013:438:452-6.

32. Ruiperez V, Darios F, Davletov B. Alpha-synuclein, lipids and Parkinson's disease. Prog Lipid Res. 2010;49:420-8.

33. Wuerker RB. Lipid droplet and microfilaments in a neuron. Tissue Cell. 1969; 1:645-51

34. Liu L, Zhang K, Sandoval H, Yamamoto S, Jaiswal M, Sanz E, Li Z, Hui J, Graham BH, Quintana A, Bellen HJ. Glial lipid droplets and ROS induced by mitochondrial defects promote neurodegeneration. Cell. 2015;160:177-90,

35. Farese RV Jr, Walther TC. Lipid droplets finally get a little R-E-S-P-E-C-T. Cell. 2009;139:855-60.

36. Lai YC, Kondapalli C, Lehneck R, Procter JB, Dill BD, Woodroof HI, Gourlay R, Peggie M, Macartney TJ, Corti O, et al. Phosphoproteomic screening identifies Rab GTPases as novel downstream targets of PINK1. EMBO J. 2015;34:2840-61.

37. Huang C, Mattis P, Perrine K, Brown N, Dhawan V, Eidelberg D. Metabolic abnormalities associated with mild cognitive impairment in Parkinson disease. Neurology. 2008;70:1470-7.

38. Kim KY, Stevens MV, Akter MH, Rusk SE, Huang RJ, Cohen A, Noguchi A, Springer D, Bocharov AV, Eggerman TL, et al. Parkin is a lipid-responsive regulator of fat uptake in mice and mutant human cells. J Clin Invest. 2011;121:3701-12

\section{Submit your next manuscript to BioMed Central} and we will help you at every step:

- We accept pre-submission inquiries

- Our selector tool helps you to find the most relevant journal

- We provide round the clock customer support

- Convenient online submission

- Thorough peer review

- Inclusion in PubMed and all major indexing services

- Maximum visibility for your research

Submit your manuscript at www.biomedcentral.com/submit 